\section{Categorization and discrimination of human and non-human primate affective vocalizations: investigation of the frontal cortex activity through fNIRS.}

${ }^{1}$ Department of Psychology and Educational Sciences and Swiss Center for Affective Sciences, University of Geneva, Geneva, Switzerland;

2 Department of Psychology, Durham University, Durham, United Kingdom;

${ }^{ \pm}$joint co-senior authors

*Correspondence to: Coralie.Debracque@unige.ch; Swiss Center for Affective Sciences, Campus Biotech, CISA - University of Geneva, Chemin des Mines 9, 1202, Geneva, Switzerland; tel: $+41223790889$

(1)

6

17

8

9

0

1

2

3

4

5

6

7

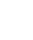

0




\section{Abstract}

Many species, including humans and non-human primates, react differently to threatening or pleasant situations. Because of its adaptiveness, recognizing affective signals is likely to be reflected in a capability of modern humans to recognize other closely related species' call content. However, at both behavioural and neural levels, only few studies have used a comparative approach to understand affective decoding processes in humans, particularly with respect to affective vocalizations. Previous research in neuroscience about the recognition of human affective vocalizations has shown the critical involvement of temporal and frontal regions. In particular, frontal regions have been reported as crucial in the explicit decoding of vocal emotions especially in different task complexity such as discrimination or categorization. The aim of this study using functional Near Infrared Spectroscopy (fNIRS) was to specifically investigate the neural activity of the inferior frontal cortex pars triangularis $\left(\mathrm{IFG}_{\text {tri }}\right.$ ) and the prefrontal cortex (PFC) underlying categorization (A versus B) and discrimination (A versus non-A) mechanisms of positive and negative affects in human, great apes (chimpanzee and bonobo), and monkey (rhesus macaque) vocalizations. We also analysed participants' behavioural responses and correlated them with the recorded frontal activations. While performing the tasks, fNIRS data revealed a clear distinction between the two frontal regions, with a general positive activation of $\mathrm{IFG}_{\text {tri }}$ compared to a decrease of PFC activity. We also found a modulation of IFG $_{\text {tri }}$ and PFC activations depending on both the species considered and on task complexity; with generally more activity in the $\mathrm{IFG}_{\text {tri }}$ during discrimination compared to categorization, and a more intense decrease of the PFC in categorization compared to discrimination. Behaviourally, participants recognized almost all affective cues in all species vocalizations at above chance levels in the discrimination task (except for threatening bonobo calls). For categorization, they mostly correctly identified at levels significantly above chance affective contents in human and great ape vocalizations but not in macaque calls. Overall, these findings support the hypothesis of a pre-human origin of affective recognition processing inherited from our common ancestor with other great apes and processed in the frontal cortex. Our results also highlight behavioural differences related to task complexity, i.e. between categorization and discrimination processes, and the differential involvement of the

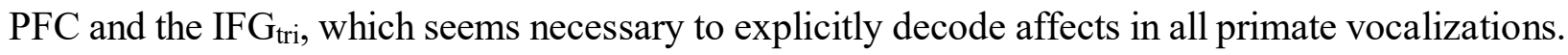




\section{Introduction}

Human life is made of choices, especially in the social domain. How we should react to threatening or joyful voices expressed by others conditions how we thrive in a given society. While usually associated with irrational choices, emotions are in fact essential to guide cognitive processes to enable adaptive responses to the environment (Brosch et al., 2013). Over the last three decades, researchers in psychology (for a review, see Lerner, Li, Valdesolo, \& Kassam, 2015) and neurosciences (for a review, see Phelps, Lempert, \& Sokol-Hessner, 2014) have investigated the impact of emotions on decision-making processes. Far from being only limited to humans, there is also a deep evolutionary origin to such recognition mechanisms. Allowing animal species to evaluate social motivations of others (Albuquerque et al., 2016) and then to react adaptively to a pleasant or a dangerous situation (Mendl \& Paul, 2020), these recognition mechanisms are crucial for the fitness of individuals (Anderson \& Adolphs, 2014; Filippi et al., 2017). In fact, perhaps even more importantly than for our own species (Homo sapiens), to correctly identify an affective signal in vocalizations is often a matter of life or death in the animal kingdom. For example, research on non-human primates (from henceforth, primates), our closest relatives, have demonstrated the capacity of chimpanzees to distinguish between different kinds of calls as function of the severities of aggression (Slocombe et al., 2009). Similar results have been found in other primates, with Gouzoules reporting the abilities of macaques to differentiate the seriousness of an agonistic interaction while listening to the victim's calls (Gouzoules, 1984).

Recent research in humans on these recognition mechanisms has emphasized the role of available sensory information as well as the different levels of complexity involved in the process during which a human makes a decision among several options (de Lange \& Fritsche, 2017). In particular, perceptual decision-making involves processing sensory information, which are evaluated and integrated according to the goal and the internal state of an individual but also depending on the possible number of choices (Hauser \& Salinas, 2014). An important aspect of this research is to investigate the cerebral basis of such recognition. However, neuroscience studies have mainly focused on the visual domain. Therefore, the neural bases of perceptual decision-making using affective auditory information remain to be investigated.

Until now, functional Magnetic Resonance Imaging (fMRI) studies involving explicit recognition of affective cues in voices have emphasized the role of frontal regions, such as the inferior frontal cortex (IFG). For instance, Brück and colleagues have revealed a stronger activation in the IFG when the participants were explicitly decoding emotional prosody as compared to identifying phonetic or semantic aspects of speech (Brück et al., 2011). These results are in line with previous research showing a key role of the IFG in affective prosody decoding (Ethofer et al., 2006; Wildgruber et al., 2009). Furthermore, recent findings have highlighted the role of the IFG in the complexity of perceptual decision-making. The categorization (unbiased choice, 'A vs B') or the discrimination (biased choice, 'A vs non-A') of affective cues in voices indeed involves different subparts of the IFG, with the involvement of the pars triangularis (IFG $\mathrm{tri}_{\text {tri }}$ ) for discrimination and the involvement of the pars opercularis ( $\mathrm{IFG}_{\mathrm{oper}}$ for categorization respectively (Dricu et al., 2017). 
Unlike IFG, the role of the prefrontal cortex (PFC), well-known for its involvement in decisionmaking (e.g. Brosch et al., 2013; Damasio, 1996), remains poorly explored in regards to the vocal decoding of emotions. Yet, the emergence of functional Near Infrared Spectroscopy (fNIRS), a non-invasive technique to study the brain hemodynamic (Boas et al., 2014) using the principle of tissue transillumination (Bright, 1831), may shed new lights on these processes. Indeed, fNIRS studies have investigated the role of PFC in emotional processing, highlighting its role in emotion regulation (Glotzbach et al., 2011) and emotion induction (Matsuo et al., 2003; Ohtani et al., 2005; Yang et al., 2007). Interestingly, recent fNIRS studies pointed out the roles of both PFC and IFG in the vocal decoding of emotions. For instance, Zhang and colleagues reported a strong involvement of the human PFC and IFG during the discrimination of affective voices (Zhang et al., 2018). Similarly, Gruber and colleagues highlighted the modulation of IFG activity depending on the categorization or the discrimination of affects in auditory stimuli (Gruber et al., 2020). Hence, more investigations on PFC and IFG activations are necessary to improve our knowledge of affective decoding. Moreover, the fNIRS methodology seems particularly adapted to the exploration of frontal regions in decision-making and emotional paradigms.

Interestingly, anatomical structures (Petrides \& Pandya, 2002; Rolls, 2004) and functions of the IFG and PFC in decision-making, auditory and affective processing are shared by most primate species, (e.g. macaques - Macaca mulatta; see Barbas, 2000; Barbas et al., 2011; Binder et al., 2004; Davidson, 1992; Frühholz \& Grandjean, 2013; Kambara et al., 2018; LeDoux, 2012). In addition, as members of the Hominidae clade, which appeared between 13 and 18 million years ago (Perelman et al., 2011), modern humans share with the other living great apes (chimpanzees Pan troglodytes, bonobos - Pan Paniscus, gorillas - Gorilla subs, and orangutans - Pongo subs) a large frontal cortex (Semendeferi et al., 2002). Overall, the fact that both humans and non-human primate species are able to identify correctly affective cues in conspecific vocalizations allowing them to use available information to make their choices; and that there is an anatomic and potentially functional convergence of the IFG and PFC across primate species, suggest that a comparative approach is particularly of interest to investigate the current role of these frontal regions in the human recognition of vocal emotions. Such approach may rely on primate calls beyond human vocalizations to uncover the evolutionary of human evaluation processes.

Yet, only a few studies have used a comparative approach to understand affective decoding mechanisms in humans using primate vocalizations. These studies have revealed at both cerebral and behavioural levels promising results highlighting the importance of the phylogenetic proximity. For example, researchers emphasized the role of the right IFG and the right orbitofrontal cortex (OFC), part of the PFC regions, in the human ability to correctly discriminate agonistic or affiliative contents in chimpanzee screams only (Belin, Fecteau, et al., 2008; Fritz et al., 2018). Nevertheless, Linnankoski and colleagues have shown the abilities of human adults and infants to recognize affective cues in macaque vocalizations using a categorization paradigm (Linnankoski et al., 1994). This last result points out the difference of complexity between the discrimination and categorization tasks in humans, even if the affective recognition is related to primate vocalizations. Overall, more controlled investigations in this domain are thus needed (Gruber \& Grandjean, 2017). 
Considering the paucity of neuroscientific studies adopting a comparative approach, the aim of the present study was to test the following questions using fNIRS: how are the human IFG and PFC regions involved in the explicit decoding of emotions contained in primate vocalizations? Is phylogenetic proximity a key for a better understanding of such processes? How does task complexity modulate the brain and behavioural responses across species and affect? To do so, we investigated human affective recognition processing in human and other primate vocalizations using cerebral and behavioural data. The participants performed categorization and discrimination tasks on affective contents (agonistic versus affiliative) in human, great apes (chimpanzee, bonobo) and monkey (rhesus macaque) vocalizations while their brain activity was recorded using fNIRS. We predicted that: i) according to the cognitive complexity hypothesis, the categorization task should involve more activations in the IFG and PFC than discrimination; ii) if a phylogenetic effect was at play, IFG and PFC would be modulated differently across human, great apes and monkey vocalizations; and iii) if frontal regions are necessary to cross-taxa recognition of affects, neural activity in the IFG and PFC should be related to the participants' performances.

\section{1}

172

173

174

175

176

177

178

179

180

181

182

183

184

185

186

187

188

189

190

191

192

193

\section{Material \& Methods}

\section{Participants}

Thirty healthy volunteers (12 males; mean age 25.06 years, $\mathrm{SD}=5.09$, age range $20-36$ ) took part in the experiment. The participants reported normal hearing abilities and normal or corrected-tonormal vision. No participant presented a neurological or psychiatric history, or a hearing impairment. All participants gave informed and written consent for their participation in accordance with the ethical and data security guidelines of the University of Geneva. The study was approved by the Ethics Cantonal Commission for Research of the Canton of Geneva, Switzerland (CCER).

\section{Vocalizations}

Ninety-six vocalizations of four primate species (human, chimpanzee, bonobo, rhesus macaque) in agonistic and affiliative contexts were used as stimuli. The human voices obtained from the Montreal Affective Voices (Belin, Fillion-Bilodeau, et al., 2008) were denoted as expressing a happy, angry or fearful affect (non-linguistic affective bursts) produced by two male and two female actors.

Vocalizations in corresponding contexts were selected for chimpanzee, bonobo and rhesus macaque species under the form of affiliative calls (food grunts), threatening calls (aggressor in agonistic context) and distress calls (victim in agonistic context). For each species, 24 stimuli were selected containing single calls or call sequences produced by 6 to 8 different individuals in their social environment.

All vocal stimuli were standardized to 750 milliseconds using PRAAT (www.praat.org) but were not normalized in order to preserve the naturalness of the sounds (Ferdenzi et al., 2013). 
fNIRS data were acquired using the Octamon device (Artinis Medical Systems B.V., Elst, The Netherlands) at $10 \mathrm{~Hz}$ with 6 transmitters and 2 receivers (wavelengths of $\pm 760 \mathrm{~nm}$ and $\pm 850 \mathrm{~nm}$ ) with an inter-distance probes at $3.5 \mathrm{~cm}$. The headband holding the 8 channels was placed identically for all participants according to the 10-20 electroencephalogram (EEG) system (Jasper, 1958; Okamoto et al., 2004) by using the FPZ axis as landmark (see Figure 1). The probe locations into the Montreal Neurological Institute (MNI) space were estimated using the 3D coordinates extracted from 32 healthy participants (Vergotte et al., 2018). Hence, the channels 1, 2, 7 and 8 were located on $\mathrm{IFG}_{\text {tri }}$ and the channels 3, 4, 5 and 6 on the PFC.

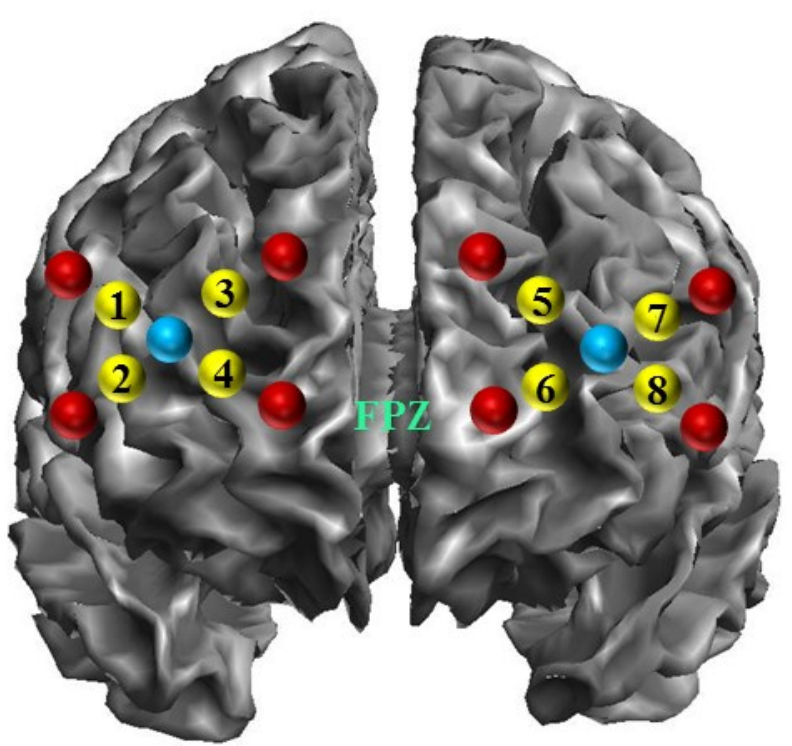

Figure 1: Probe locations into the MNI space by using SPM12 software implemented in MatLab R2018b (www.fil.ion.ucl.ac.uk/spm/). Red and blue dots indicate transmitters and receivers' positions respectively. Yellow dots indicate the channel numbers.

\section{Experimental procedure}

Seated comfortably in front of a computer, participants listened to the vocalizations played binaurally using Seinnheiser headphones at $70 \mathrm{~dB}$ SPL. Each of the 96 stimuli was repeated nine times across six separate blocks leading to 864 trials following a randomization process. The overall experiment was structured in various layers (Figure 2). Testing blocks were task-specific, with participants having to either perform a categorization task (A versus B) or a discrimination task (A versus non-A) in a single block. Participants completed three categorization blocks and three discrimination blocks, resulting in six blocks in total. Each block was made of 12 miniblocks, each separated by a break of 10 seconds. These mini-blocks comprised one unique miniblock per species (human, chimpanzee, bonobo and rhesus macaque), each mini-block repeated 3 times. Within each mini-block were 12 trials, containing four vocalisations from all three affective contexts (affiliative/happy; threatening/anger; fear) produced by a single species. The blocks, mini-blocks and stimuli were pseudo-randomly assigned for each participant to avoid more than two consecutive blocks, mini-blocks and stimuli from the same category. 
At the beginning of each block, participants were instructed to identify the affective content of the vocalizations using a keyboard. For instance, the instructions for the categorization task could be “Affiliative - press M or Threatening - press Z or Distress - press space bar". Similarly, the instructions for discrimination could be "Affiliative - press $Z$ or other affect - press M". The pressed keys were randomly assigned across blocks and participants. The participants had to press the key during the 2-second intervals (jittering of $400 \mathrm{~ms}$ ) between each stimulus. If the participant did not respond during this interval, the next stimulus followed automatically.

Figure 2: Structure of the experiment, with each of the six blocks made of 12 mini-blocks, which in turn comprised 12 individual trials.

\section{Statistical analysis}

\section{Behavioural data}

Raw behavioural data from all participants were analysed using Generalized Linear Mixed Model (GLMM) fitted by Restricted Maximum Likelihood (REML) on R.studio (Team, 2020) with the "bobyqa" function (optimization by quadratic approximation with a set maximum of 1'000'000 iterations) and the link "logit" for a standard logistic distribution or errors and a binomial error distribution (correct answer - 1 or not - 0) of the package Lme4 (Bates et al., 2015). The following three factors and their interactions were included: Species (human, chimpanzee, bonobo, and rhesus macaque), Tasks (categorization - CAT and discrimination - DIS), and Affects (affiliative, threat, and distress). Participant IDs and order of the blocks were used as random factors. In order to test our hypotheses regarding the phylogenetic distance and the task complexity on participants' performances we compared, using contrasts, the differences between Species and Affects within the categorization and the discrimination tasks. These contrasts were corrected with Bonferroni correction $\left(\mathrm{P}_{\text {corrected }}=.05 /\right.$ number of tests $\left.=.05 / 24=.002\right)$. Similarly, the participants' reaction time (correct answers only) were analysed using a GLMM with a Gaussian distribution with the same contrasts and analysis as for accuracy. The present paper focusing on the investigation of recognition mechanisms, not attentional processes, results for reaction times are reported in supplementary material. 
Ten participants out of 30 were excluded from the dataset due to poor signal quality (large number of artefacts after filtering) or missing fNIRS data. A total of 20 participants were thus analysed in this study, in line with previous power analyses in fMRI (Desmond \& Glover, 2002) and research using fNIRS to assess emotional processing in frontal areas (for a review, see Bendall et al., 2016). We performed on all channels the first level analysis with MatLab 2018b (Mathwortks, Natick, MA) using the SPM_fNIRS toolbox (Tak, Uga, Flandin, Dan, \& Penny, 2016; https://www.nitrc.org/projects/spm fnirs/) and homemade scripts. Haemoglobin conversion and temporal pre-processing of $\mathrm{O}_{2} \mathrm{Hb}$ was made using the following procedure:

1. Haemoglobin concentration changes were calculated with the modified Beer-Lambert law (Delpy et al., 1988);

2. Motion artefacts were reduced using the movement artefact reduction algorithm (MARA Scholkmann et al., 2010) based on moving standard deviation and spline interpolation;

3. Low frequency confound were reduced using a high-pass filter based on a discrete cosine transform set with a cut-off frequency of $1 / 64 \mathrm{~Hz}$ (Friston et al., 2000);

4. Physiological and high frequency noise such vasomotion or heart beats usually found in extra-cerebral blood flow were removed using a low-pass filter based on the hemodynamic response function (HRF - Friston et al., 2000).

5. $\mathrm{O}_{2} \mathrm{Hb}$ concentration changes were averaged between 4 and 12 seconds post stimulus onset on each trial to include the maximum peak amplitude of the HRF observed across participants. As for fMRI imaging, this method of analysis taking into account the slow hemodynamic time course of brain activity is in line with previous literature using auditory stimuli in fNIRS (e.g. Lloyd-Fox et al., 2014).

The second level analysis was performed on R. studio using GLMM fitted by REML with the factors: Species (human, chimpanzee, bonobo, rhesus macaque), Tasks (categorization versus discrimination), Affects (affiliative, threatening, distressful), as well as their interactions as fixed factors, and participant IDs and block orders as random factors for the right and left IFG $\mathrm{tri}_{\text {tri }}$ and PFC.

\section{Interaction between participants' performance and brain Oxyhemoglobin $\left(\mathrm{O}_{2} \mathrm{Hb}\right)$ changes}

To test whether the IFG $_{\text {tri }}$ and PFC activations facilitated the participants' affective recognition, we used fNIRS data as continuous predictors in GLMM analysis performed on R. studio for accuracy. To perform this statistical interaction, we only used accuracy from the twenty participants included in fNIRS analyses. The GLMM fitted by REML included Species (human, chimpanzee, bonobo and rhesus macaque), Tasks (discrimination and categorization), Affects (threat, distress and affiliative), as fixed factors, fNIRS data from the right and left IFG $\mathrm{tri}_{\text {tri }}$ and PFC as continuous predictors, and participant IDs as a random factor. To assess the variance explained by the phylogeny as well within the frontal activation, we tested all slopes with the following contrast: human vs [great apes (chimpanzee and bonobo)] vs rhesus macaque. 


\section{Results}

\section{Accuracy}

We investigated how the perceptual decision-making complexity influenced the ability of human participants to recognize affective contents in phylogenetically close or distant primate species (see Figure 3).

Hence, participants were significantly above chance $(>50 \%$ in discrimination; $>33 \%$ in categorization) for most of the affective cues in great ape vocalizations (threatening bonobo calls excluded - see Table 1). Yet, they were unable to do so for threatening macaque calls in the discrimination task and all affective vocalizations expressed by this species in the categorisation one. Moreover, human participants were better at discriminating and then categorizing human voices $($ threat $=$ DIS 76\%; CAT 60\%, distress $=$ DIS 77\%; CAT 68\%, affiliative $=$ DIS 83\%; CAT 69\%), chimpanzee distress (DIS 65\%; CAT 50\%) and threatening (DIS 63\%; CAT 50\%) vocalizations, followed by distress and affiliative calls expressed by bonobos (DIS 62\%; CAT 46\% for both) and macaques in the discrimination task (62\%).

306

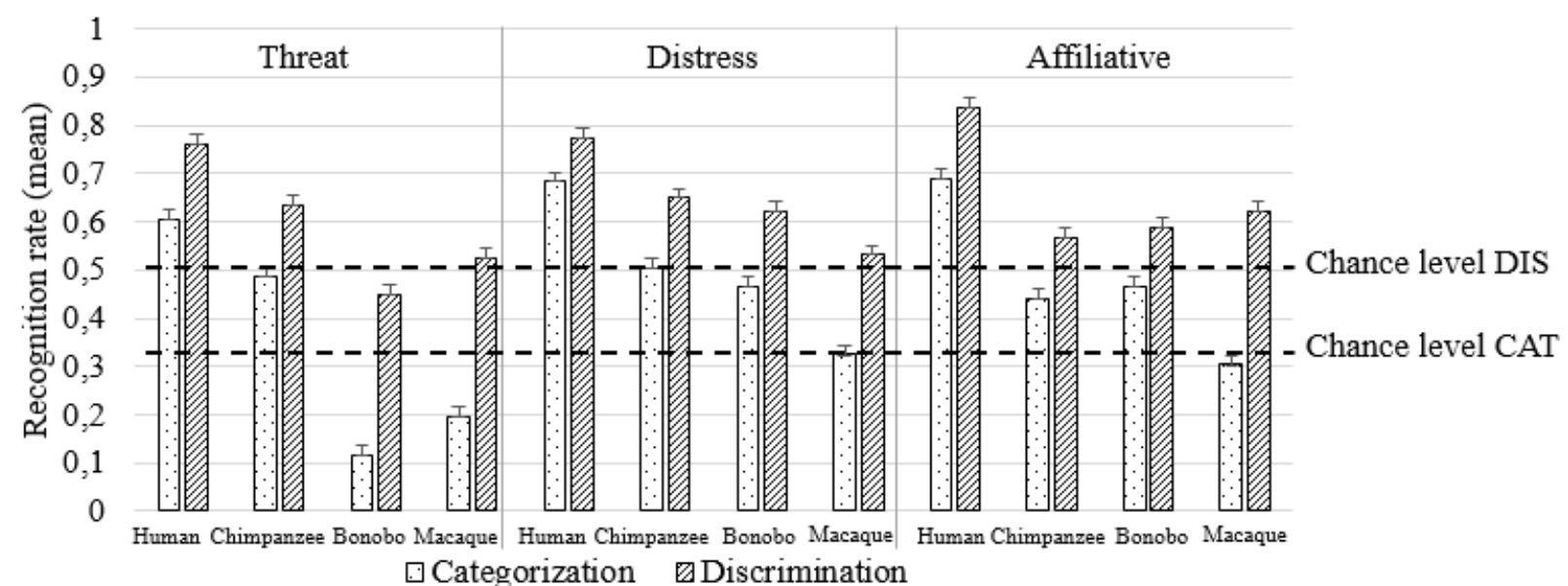

Figure 3: Mean and SE of human recognition of primate affective vocalizations for categorization (CAT) and discrimination (DIS) tasks and the different kinds of affective vocalizations. All contrasts were significant within each condition after Bonferroni correction with $\mathrm{P}_{\text {corrected }}=$ $.05 / 24=.002$, excluding the following contrasts: chimpanzee $v s$ macaque and bonobo $v s$ macaque for affiliative cues and bonobo vs macaque for threatening contents in discrimination task (see supplementary material Table 1). 
Table 1: Summary of the one sample t-test analyses against chance level. Recognition performance above chance ( $>33 \%$ categorisation and $>50 \%$ discrimination) are written in bold. $* * * \mathrm{p}<.001$, * $\mathrm{p}<.05$.

\begin{tabular}{|l|l|l|l|l|l|l|}
\hline \multicolumn{4}{c|}{ Categorization } & \multicolumn{3}{c|}{ Discrimination } \\
\hline & Threat & Distress & Affiliative & Threat & Distress & Affiliative \\
\hline Bonobo & $-22.19^{* * *}$ & $\mathbf{8 . 9 5}^{* * *}$ & $\mathbf{8 . 9 6}^{* * *}$ & $-3.44^{* * *}$ & $\mathbf{8 . 2 6}^{* * *}$ & $\mathbf{5 . 9}^{* * *}$ \\
\hline Chimpanzee & $\mathbf{1 0 . 2 7}^{* * *}$ & $\mathbf{1 1 . 4 1}^{* * *}$ & $\mathbf{7 . 3 5}^{* * *}$ & $\mathbf{9 . 1 5}^{* * *}$ & $\mathbf{1 0 . 1 9}^{* * *}$ & $\mathbf{4 . 5 1}^{* * *}$ \\
\hline Human & $\mathbf{1 8 . 4 7} * * *$ & $\mathbf{2 5 . 0 7}^{* * *}$ & $\mathbf{2 5 . 6 7}^{* * *}$ & $\mathbf{1 9 . 9 2}^{* * *}$ & $\mathbf{2 1 . 3 3}^{* * *}$ & $\mathbf{2 9 . 5 1} * * *$ \\
\hline Macaque & $-11.15^{* * *}$ & -0.41 & -1.93 & 1.53 & $\mathbf{2 . 0 2}^{*}$ & $\mathbf{8 . 1 3}^{* * *}$ \\
\hline
\end{tabular}

\section{fNIRS data}

A significant main effect was found for the factor Tasks in the right $\operatorname{IFG}_{\text {tri }}\left(\chi^{2}(1)=14.27, p<.001\right)$; left $\operatorname{IFG}_{\text {tri }}\left(\chi^{2}(1)=3.89, \mathrm{p}<.05\right)$; right PFC $\left(\chi^{2}(1)=107.32, \mathrm{p}<0.001\right)$ and left $\operatorname{PFC}\left(\chi^{2}(1)=90.83\right.$, $\mathrm{p}<.001)$ revealing more $\mathrm{O} 2 \mathrm{Hb}$ concentration changes for the discrimination compared to the categorization task for all ROIs (see Figure 4). Note that none of the interactions with the factors Affects and Species reached significance.

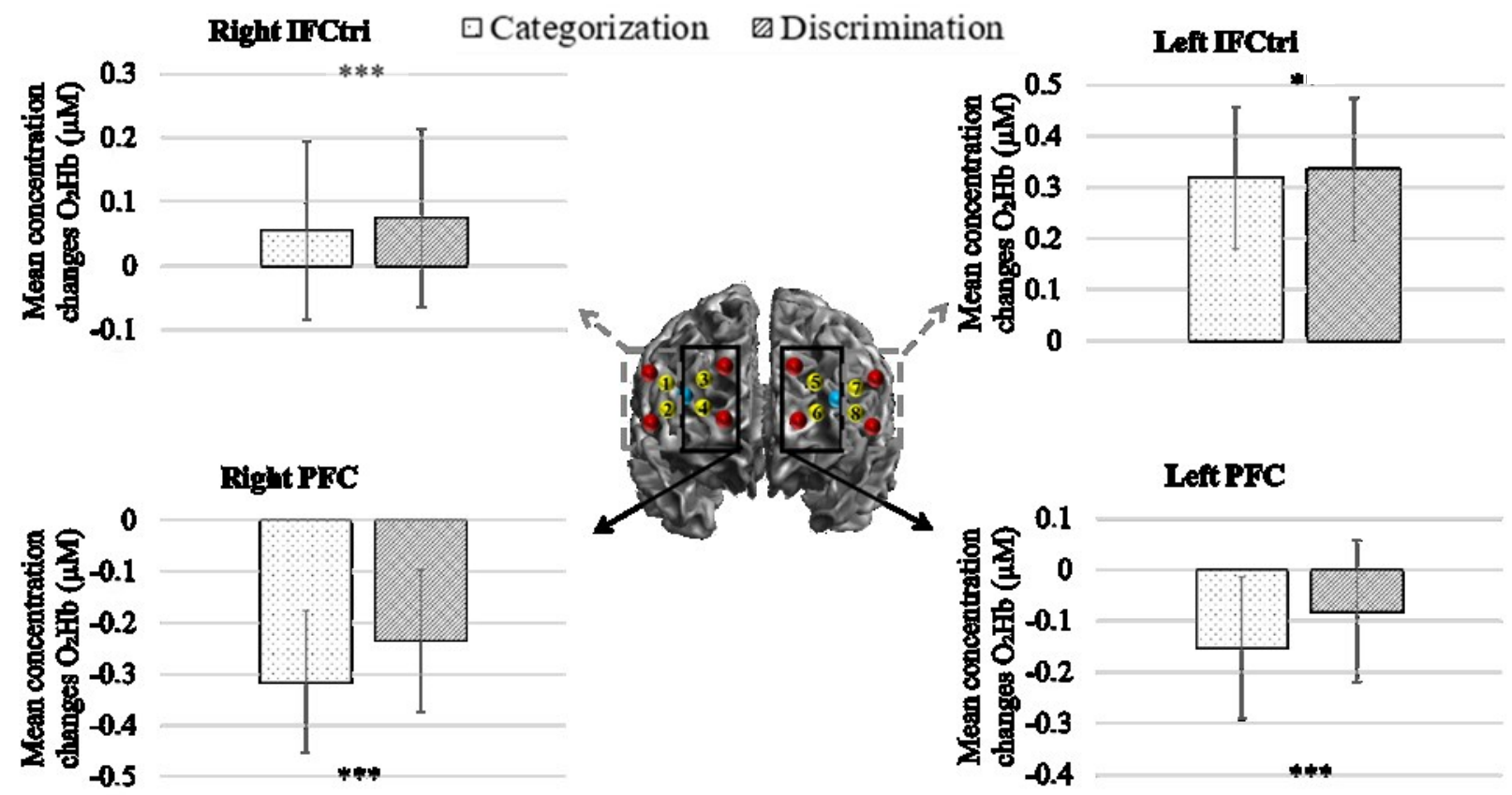

Figure 4: Mean and SE of concentration changes of $\mathrm{O}_{2} \mathrm{Hb}(\mu \mathrm{M})$ in right and left $\mathrm{PFC}$ and $\mathrm{IFG}_{\text {tri }}$ during the categorization and the discrimination tasks by human participants of primate affective vocalizations. $* * * \mathrm{p}<.001, * \mathrm{p}<.05$. 


\section{Interaction between participants' performance and brain $\mathrm{O}_{2} \mathrm{Hb}$ changes}

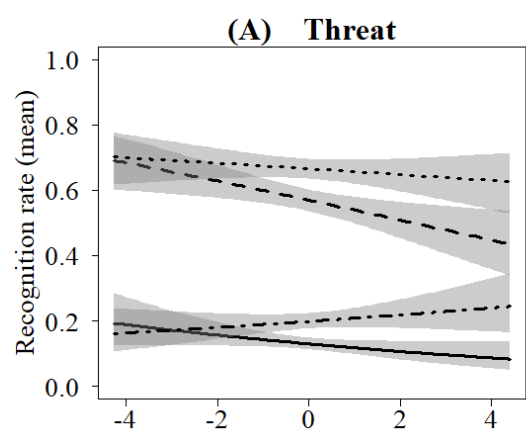

(B)

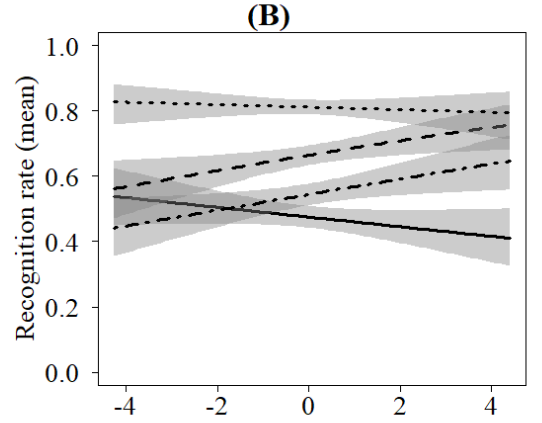

360

361

362

363 $.001)$. Figure 3. regions.

All factors (Tasks, Species and Affects) with the fNIRS data of the right and left IFG tri and PFC as continuous predictors contributed to a significant three-way interaction $\left(\chi^{2}(24)=202,28 \mathrm{p}<\right.$

Within this model, we then assessed how the affective contents modulated IFG $_{\text {tri }}$ and PFC activity across species vocalizations during the categorization or discrimination tasks. For this purpose, we investigated whether the participants' accuracy and the related fNIRS data were positively, negatively or not correlated for each Species and ROIs within the Affects and Tasks factors using odd-ratio summarized in Table 2. In particular we tested whether phylogenetic proximity facilitated the recognition of Affect. We found for both the IFG $\mathrm{tri}_{\text {and }}$ PFC that contrasts between humans vs [great apes (chimpanzees and bonobos)] vs rhesus macaques within each Affect and Task were significant at $\mathrm{p}<.001$ (see supplementary material Table 3 ). Note that because we found similar patterns of performances between PFC and $\mathrm{IFG}_{\mathrm{tri}}$, for more clarity, we will only describe the results for IFG $_{\text {tri }}$ here (see Figure 5). Results for PFC are reported in supplementary material

Hence, participants better discriminated agonistic (threat and distress) chimpanzee calls when the concentration changes of $\mathrm{O}_{2} \mathrm{Hb}$ increased in $\mathrm{IFG}_{\text {tri }}$ and PFC. At the opposite, during the categorization task, the correct identification of all types of chimpanzee calls as well as affiliative macaque and agonistic bonobo vocalizations were associated with a decrease of activity in frontal
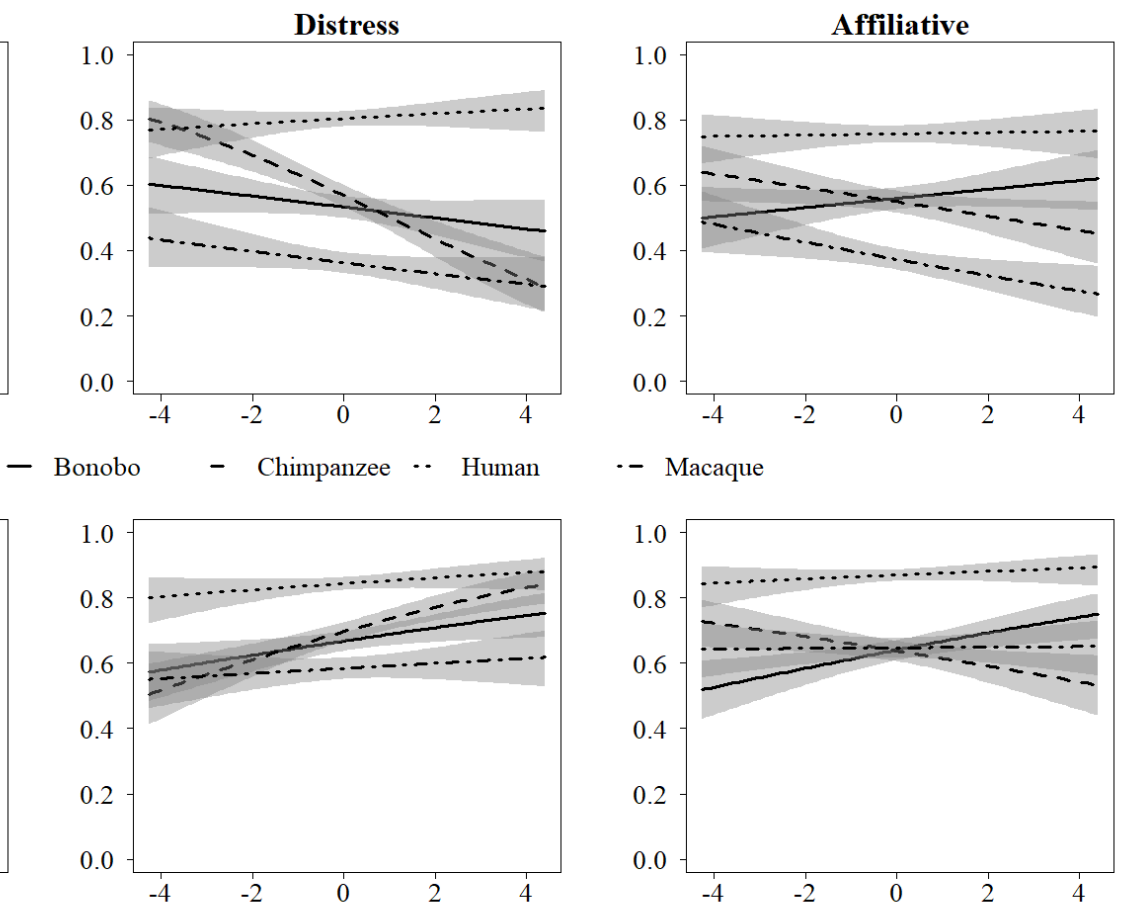

$\mathrm{O} 2 \mathrm{Hb}$ changes in IFCtri $(\log \mu \mathrm{M})$

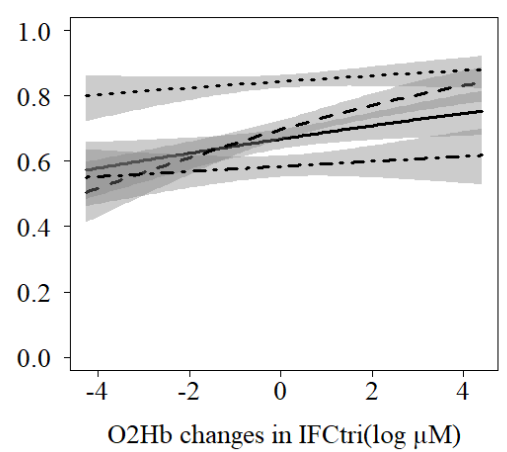

Figure 5: Interaction between participants' accuracy and $\mathrm{O}_{2} \mathrm{Hb}$ concentration changes in IFG $\mathrm{G}_{\text {tri }}$ within each affect and species for (A) categorization and (B) discrimination. Confidence interval at 0.95. Figures were made on R.studio using the package Visreg (Breheny \& Burchett, 2017). 
Table 2: Summary of the odds ratio and p-values testing the statistical significance and the direction of logistic regression slopes from the three-way interaction. The odds ratio quantifies the strength of the association between two factors. If the slope is significant and odds ratio $<1$, factors are negatively correlated (written in bold); if the slope is significant and odds ratio $>1$, factors are positively correlated (written in bold italic). $* * \mathrm{p}<.01, * \mathrm{p}<.05$.

\begin{tabular}{|l|l|l|l|l|l|l|}
\hline \multicolumn{3}{|c|}{ Categorization } & \multicolumn{3}{c|}{ Discrimination } \\
\hline & Threat & Distress & Affiliative & Threat & Distress & Affiliative \\
\hline Bonobo & $\mathbf{0 . 8 4}^{*}$ & $\mathbf{0 . 8 8}^{*}$ & 1.06 & 0.99 & 1.1 & 1.06 \\
\hline Chimpanzee & $\mathbf{0 . 7 8}^{*}$ & $\mathbf{0 . 6 9}^{*}$ & $\mathbf{0 . 8 6}^{*}$ & $\mathbf{1 . 2 8}^{*}$ & $\mathbf{1 . 4 4}^{* *}$ & 0.93 \\
\hline Human & 1.02 & 1.13 & 1.11 & 0.98 & 0.89 & 1.02 \\
\hline Macaque & 1.07 & 0.94 & $\mathbf{0 . 8 5}^{*}$ & 0.93 & 0.9 & 1.05 \\
\hline
\end{tabular}

\section{Discussion}

The present study emphasized the different levels of complexity in decision-making processes underlying the human recognition of affects in human and non-human primate vocalizations. In particular, we demonstrated that the left $\mathrm{IFG}_{\text {tri }}$ and the right PFC were strongly involved in the discrimination task compared to the categorization one.

Interestingly, and perhaps, contradictorily, we initially expected more activation in $\mathrm{IFG}_{\mathrm{tri}}$ for the categorization task (unbiased choice) because of the existing literature on human affective voices (Dricu et al., 2017; Gruber et al., 2020). However, taking into account our behavioural results showing higher recognition performances in discrimination compared to categorization, more activity in IFG $_{\text {tri }}$ appears to be required to enable participants to perform better during the discrimination of primate vocalizations. At the opposite, in line with the cognitive complexity hypothesis, analyses for PFC revealed a stronger deactivation in the categorization task. We could link these last findings to the changes in regional cerebral blood flow. Indeed, Matsukawa and collaborators showed that during the passive viewing of emotional videos, the activity of PFC decreased in correlation to the reduction of facial skin blood flow (Matsukawa et al., 2018). Interestingly, these authors suggested that PFC activity might elicit an autonomic reaction with a vasoconstriction or a vasodilatation of cutaneous vessels. In the same line, George and collaborators demonstrated a stronger decrease of activity in right PFC during the viewing of pleasant pictures, also relying on a reduction of the frontal blood flow (George et al., 1995). A possibility is thus to extend the results of these visual studies to a decrease of activity in PFC regions during affective auditory processing.

Overall, our results highlight the distinct roles of the $\mathrm{IFG}_{\text {tri }}$ and the $\mathrm{PFC}$ in evaluative judgment and decision task in affective primate calls recognition (see Schirmer \& Kotz, 2006; Wagner \& Watson, 2010 for humans).

Was human recognition influenced by the affects and/or the species that expressed the vocalizations? We did find an influence of these factors on behavioural responses and the interaction between participants' performances and frontal activations. In fact, we demonstrated that the correct categorization of agonistic cues in bonobo and chimpanzee vocalizations elicited a significant decrease of activity in the $\mathrm{IFG}_{\text {tri }}$ and the PFC. These results might be related to an 
inhibition process enabling participants to reduce a high level of stress elicited by agonistic calls, i.e. automatic regulation. Frontal regions are indeed the most sensitive brain areas to stress exposure (Arnsten, 2009). Interestingly, a decrease of activation in frontal regions was also associated to better performance in the categorization task for affiliative chimpanzee and macaque vocalizations. On the contrary, in the discrimination task, agonistic chimpanzee screams were better identified when the level of activity in IFG $_{\text {tri }}$ and PFC increased. These results highlight the involvement of distinct mechanisms between the categorization and discrimination tasks in crosstaxa recognition. For instance, possible inhibition processes elicited by agonistic cues would rely on a decrease of activations in frontal regions for the simple choice between A versus non-A; while in categorisation (unbiased choice), similar inhibition mechanisms would require an enhancement of activity in IFG $_{\text {tri }}$ and PFC.

The general absence of interaction between frontal activations and behaviours for human voices might be explained by three different mechanisms. First, for humans, because affective voices in our modern human societies are everywhere (Belin, 2006), the correct recognition of affects may not necessary involve particular frontal activations due to the human expertise in human voice processing. Second, the involvement of IFG has often been demonstrated in the literature for the recognition of emotional voices contrasted with neutral ones (e.g. Frühholz et al., 2012; Frühholz \& Grandjean, 2013; Gruber et al., 2020; Sander et al., 2005; Zhang et al., 2018). Yet, in our study, we did not include such stimuli, comparing cerebral activations across the affective contents. This difference in our experimental paradigm may have led to the absence of interaction between the hemodynamic response in the frontal regions and the emotional recognition in human voices. Third, encompassing three neuroanatomical and functional subparts: pars triangularis, pars orbitalis and pars opercularis (Cai \& Leung, 2011), IFG $\mathrm{G}_{\text {tri }}$ would possibly requires the recognition of infrequent vocalizations expressed by evolutionary close species to be modulated. Following this, the phylogenetic gap of 25-33 million between rhesus macaque and the Hominidae branch might explain the lack of result for this monkey species. Performances on the macaque calls categorization were poor, hence the frontal activations would not help to categorize them because human participants were, at least in this experiment, unable to categorize these calls. In contrast, participants were able to categorize most affects in great ape vocalizations, to the exception of threatening bonobo calls.

Yet, such reasoning does not apply to discrimination, where the low level of cognitive complexity involved may have allowed participants to discriminate more correctly affective vocalizations of all primates, including species with larger phylogenetic distances such as macaques. Strikingly, behavioural analyses revealed that human participants were able to discriminate most of the affective cues in all species vocalizations, once again to the exception of threatening bonobo calls. We might hypothesize that specific acoustic factors in bonobo calls triggered this effect: bonobo calls have indeed a higher fundamental frequency resulting from a shorter vocal length in comparison to chimpanzees. In this species, signalling physical strength using low frequencies (e.g. Briefer, 2012; Morton, 1982) is not a sexually selected trait (Grawunder et al., 2018). This reflects in their general behaviour, with bonobos being quite different from closely related chimpanzees and overall less aggression prone: they are occasional hunters, do not have strict territories and have a developed socio-sexuality, reducing the number of aggressive conflicts (Gruber \& Clay, 2016). 
To conclude, our findings demonstrate the interplay between cerebral and behavioural processes during the recognition by humans of affective cues in primate vocalizations. Decision-making complexity, phylogeny and behaviour seem four essential markers to consider for further studies on cross-taxa recognition. Overall, we demonstrated the difference of mechanisms between the categorization and discrimination of primate affective calls at both behavioural and cerebral levels. In particular, we showed various activations in the PFC and IFG $\mathrm{Ftri}_{\text {and }}$ and their connection to the ability of humans to correctly identify affective cues in great apes and monkeys' vocalizations. Furthermore, our results highlighted the importance of the phylogenetic proximity in affective recognition processes. Finally, to our knowledge, this study is the first to: i) distinguish categorization and discrimination processes in a neuroscientific experiment with a comparative perspective, and ii) to assess the link between cross-taxa affective recognition and frontal activations in a fNIRS paradigm. We hope these new findings will contribute to a better understanding of the evolutionary origins of emotional processing and decision-making origin in human, as well as advocate for the inclusion of a broader array of auditory stimuli.

\section{Acknowledgements}

We thank Katie Slocombe very much for providing chimpanzee and macaque auditory stimuli as well as extensive comments on former versions of this preprint. We would like also to thanks Dr. Ben Meuleman for his useful support on statistical analyses. We thank the Swiss National Science foundation (SNSF) for supporting this interdisciplinary project (CR13I1_162720 / 1 - DG-TG), and the Swiss Center for Affective Sciences. ZC has received support from the ESRC-ORA (ES/S015612/1), the ERC Starting Grant (802979, and CD from the foundation Ernst and Lucie Schmidheiny. TG was additionally supported by a grant of the SNSF during the final re-writing of this article (grant PCEFP1_186832).

\section{References}

Albuquerque, N., Guo, K., Wilkinson, A., Savalli, C., Otta, E., \& Mills, D. (2016). Dogs recognize dog and human emotions. Biology Letters, 12(1), 20150883. https://doi.org/10.1098/rsbl.2015.0883

Anderson, D. J., \& Adolphs, R. (2014). A Framework for Studying Emotions Across Phylogeny. Cell, 157(1), 187-200. https://doi.org/10.1016/j.cell.2014.03.003

Arnsten, A. F. T. (2009). Stress signalling pathways that impair prefrontal cortex structure and function. Nature Reviews. Neuroscience, 10(6), 410-422. https://doi.org/10.1038/nrn2648

Bates, D., Mächler, M., Bolker, B., \& Walker, S. (2015). Fitting Linear Mixed-Effects Models Using lme4. Journal of Statistical Software, 67(1), 1-48. https://doi.org/10.18637/jss.v067.i01

Belin, P. (2006). Voice processing in human and non-human primates. Philosophical Transactions of the Royal Society B: Biological Sciences, 361(1476), 2091-2107. https://doi.org/10.1098/rstb.2006.1933 
Belin, P., Fecteau, S., Charest, I., Nicastro, N., Hauser, M. D., \& Armony, J. L. (2008). Human cerebral response to animal affective vocalizations. Proceedings. Biological Sciences, 275(1634), 473-481. https://doi.org/10.1098/rspb.2007.1460

Belin, P., Fillion-Bilodeau, S., \& Gosselin, F. (2008). The Montreal Affective Voices: A validated set of nonverbal affect bursts for research on auditory affective processing. Behavior Research Methods, 40(2), 531-539. https://doi.org/10.3758/BRM.40.2.531

Bendall, R. C. A., Eachus, P., \& Thompson, C. (2016). A Brief Review of Research Using NearInfrared Spectroscopy to Measure Activation of the Prefrontal Cortex during Emotional Processing: The Importance of Experimental Design. Frontiers in Human Neuroscience, 10, 529. https://doi.org/10.3389/fnhum.2016.00529

Boas, D. A., Elwell, C. E., Ferrari, M., \& Taga, G. (2014). Twenty years of functional near-infrared spectroscopy: Introduction for the special issue. NeuroImage, 85, 1-5. https://doi.org/10.1016/j.neuroimage.2013.11.033

Breheny, P., \& Burchett, W. (2017). Visualization of Regression Models Using visreg. The $R$ Journal, 9(2), 56. https://doi.org/10.32614/RJ-2017-046

Briefer, E. (2012). Vocal Expression of Emotions in Mammals: Mechanisms of Production and Evidence. Communication Skills. https://animalstudiesrepository.org/comski/1

Bright, R. (1831). Reports of medical cases selected with a view of illustrating the symptoms and cure of diseases by reference to morbid anatomy, case ccv "diseases of the brain and nervous system." 2(3), 431.

Brosch, T., Scherer, K., Grandjean, D., \& Sander, D. (2013). The impact of emotion on perception, attention, memory, and decision-making. Swiss Medical Weekly, 143(1920). https://doi.org/10.4414/smw.2013.13786

Brück, C., Kreifelts, B., Kaza, E., Lotze, M., \& Wildgruber, D. (2011). Impact of personality on the cerebral processing of emotional prosody. NeuroImage, 58(1), 259-268. https://doi.org/10.1016/j.neuroimage.2011.06.005

Cai, W., \& Leung, H.-C. (2011). Rule-guided executive control of response inhibition: Functional topography of the inferior frontal cortex. PloS One, 6(6), e20840. https://doi.org/10.1371/journal.pone.0020840

Damasio, A. R. (1996). The somatic marker hypothesis and the possible functions of the prefrontal cortex. Philosophical Transactions of the Royal Society of London. Series B, Biological Sciences, 351(1346), 1413-1420. https://doi.org/10.1098/rstb.1996.0125

de Lange, F. P., \& Fritsche, M. (2017). Perceptual Decision-Making: Picking the Low-Hanging Fruit? Trends in Cognitive Sciences, 21(5), 306-307. https://doi.org/10.1016/j.tics.2017.03.006

Delpy, D. T., Cope, M., van der Zee, P., Arridge, S., Wray, S., \& Wyatt, J. (1988). Estimation of optical pathlength through tissue from direct time of flight measurement. Physics in Medicine and Biology, 33(12), 1433-1442. 
Desmond, J. E., \& Glover, G. H. (2002). Estimating sample size in functional MRI (fMRI) neuroimaging studies: Statistical power analyses. Journal of Neuroscience Methods, 118(2), 115-128. https://doi.org/10.1016/s0165-0270(02)00121-8

Dricu, M., Ceravolo, L., Grandjean, D., \& Frühholz, S. (2017). Biased and unbiased perceptual decision-making on vocal emotions. Scientific Reports, 7(1), 16274. https://doi.org/10.1038/s41598-017-16594-w

Ethofer, T., Anders, S., Erb, M., Herbert, C., Wiethoff, S., Kissler, J., Grodd, W., \& Wildgruber, D. (2006). Cerebral pathways in processing of affective prosody: A dynamic causal modeling study. $\quad$ NeuroImage, $30(2), \quad 580-587$. https://doi.org/10.1016/j.neuroimage.2005.09.059

Ferdenzi, C., Patel, S., Mehu-Blantar, I., Khidasheli, M., Sander, D., \& Delplanque, S. (2013). Voice attractiveness: Influence of stimulus duration and type. Behavior Research Methods, 45(2), 405-413. https://doi.org/10.3758/s13428-012-0275-0

Filippi, P., Congdon, J. V., Hoang, J., Bowling, D. L., Reber, S. A., Pašukonis, A., Hoeschele, M., Ocklenburg, S., de Boer, B., Sturdy, C. B., Newen, A., \& Güntürkün, O. (2017). Humans recognize emotional arousal in vocalizations across all classes of terrestrial vertebrates: Evidence for acoustic universals. Proceedings of the Royal Society B: Biological Sciences, 284(1859), 20170990. https://doi.org/10.1098/rspb.2017.0990

Friston, K. J., Josephs, O., Zarahn, E., Holmes, A. P., Rouquette, S., \& Poline, J. (2000). To smooth or not to smooth? Bias and efficiency in fMRI time-series analysis. NeuroImage, 12(2), 196-208. https://doi.org/10.1006/nimg.2000.0609

Fritz, T., Mueller, K., Guha, A., Gouws, A., Levita, L., Andrews, T. J., \& Slocombe, K. E. (2018). Human behavioural discrimination of human, chimpanzee and macaque affective vocalisations is reflected by the neural response in the superior temporal sulcus. Neuropsychologia, 111, 145-150. https://doi.org/10.1016/j.neuropsychologia.2018.01.026

Frühholz, S., Ceravolo, L., \& Grandjean, D. (2012). Specific brain networks during explicit and implicit decoding of emotional prosody. Cerebral Cortex (New York, N.Y.: 1991), 22(5), 1107-1117. https://doi.org/10.1093/cercor/bhr184

Frühholz, S., \& Grandjean, D. (2013). Processing of emotional vocalizations in bilateral inferior frontal cortex. Neuroscience and Biobehavioral Reviews, 37(10 Pt 2), 2847-2855. https://doi.org/10.1016/j.neubiorev.2013.10.007

George, M. S., Ketter, T. A., Parekh, P. I., Horwitz, B., Herscovitch, P., \& Post, R. M. (1995). Brain activity during transient sadness and happiness in healthy women. The American Journal of Psychiatry, 152(3), 341-351. https://doi.org/10.1176/ajp.152.3.341

Glotzbach, E., Mühlberger, A., Gschwendtner, K., Fallgatter, A. J., Pauli, P., \& Herrmann, M. J. (2011). Prefrontal Brain Activation During Emotional Processing: A Functional Near Infrared Spectroscopy Study (fNIRS). The Open Neuroimaging Journal, 5, 33-39. https://doi.org/10.2174/1874440001105010033 
Gouzoules, S. (1984). Primate mating systems, kin associations, and cooperative behavior: Evidence for kin recognition? American Journal of Physical Anthropology, 27(S5), 99134. https://doi.org/10.1002/ajpa.1330270506

Grawunder, S., Crockford, C., Clay, Z., Kalan, A. K., Stevens, J. M. G., Stoessel, A., \& Hohmann, G. (2018). Higher fundamental frequency in bonobos is explained by larynx morphology. Current Biology: CB, 28(20), R1188-R1189. https://doi.org/10.1016/j.cub.2018.09.030

Gruber, T., \& Clay, Z. (2016). A Comparison Between Bonobos and Chimpanzees: A Review and Update. Evolutionary Anthropology: Issues, News, and Reviews, 25(5), 239-252. https://doi.org/10.1002/evan.21501

Gruber, T., Debracque, C., Ceravolo, L., Igloi, K., Marin Bosch, B., Frühholz, S., \& Grandjean, D. (2020). Human Discrimination and Categorization of Emotions in Voices: A Functional Near-Infrared Spectroscopy (fNIRS) Study. Frontiers in Neuroscience, 14. https://doi.org/10.3389/fnins.2020.00570

Gruber, T., \& Grandjean, D. M. (2017). A comparative neurological approach to emotional expressions in primate vocalizations. Neuroscience and Biobehavioral Reviews, 73, 182190.

Hauser, C. K., \& Salinas, E. (2014). Perceptual Decision Making. In D. Jaeger \& R. Jung (Eds.), Encyclopedia of Computational Neuroscience (pp. 1-21). Springer New York. https://doi.org/10.1007/978-1-4614-7320-6_317-1

Jasper, H. H. (1958). The Ten-Twenty Electrode System of the International Federation. 10, 371375.

Lerner, J. S., Li, Y., Valdesolo, P., \& Kassam, K. S. (2015). Emotion and Decision Making. Annual Review of Psychology, 66(1), 799-823. https://doi.org/10.1146/annurev-psych-010213115043

Linnankoski, I., Laakso, M., Aulanko, R., \& Leinonen, L. (1994). Recognition of emotions in macaque vocalizations by children and adults. Language \& Communication, 14(2), 183192. https://doi.org/10.1016/0271-5309(94)90012-4

Lloyd-Fox, S., Papademetriou, M., Darboe, M. K., Everdell, N. L., Wegmuller, R., Prentice, A. M., Moore, S. E., \& Elwell, C. E. (2014). Functional near infrared spectroscopy (fNIRS) to assess cognitive function in infants in rural Africa. Scientific Reports, 4, 4740. https://doi.org/10.1038/srep04740

Matsukawa, K., Asahara, R., Yoshikawa, M., \& Endo, K. (2018). Deactivation of the prefrontal cortex during exposure to pleasantly-charged emotional challenge. Scientific Reports, 8(1), 14540. https://doi.org/10.1038/s41598-018-32752-0

Matsuo, K., Kato, T., Taneichi, K., Matsumoto, A., Ohtani, T., Hamamoto, T., Yamasue, H., Sakano, Y., Sasaki, T., Sadamatsu, M., Iwanami, A., Asukai, N., \& Kato, N. (2003). Activation of the prefrontal cortex to trauma-related stimuli measured by near-infrared spectroscopy in posttraumatic stress disorder due to terrorism. Psychophysiology, 40(4), 492-500. https://doi.org/10.1111/1469-8986.00051 
Mendl, M., \& Paul, E. S. (2020). Animal affect and decision-making. Neuroscience \& Biobehavioral Reviews, 112, 144-163. https://doi.org/10.1016/j.neubiorev.2020.01.025 Morton, E. S. (1982). Grading, discreteness, redundancy, and motivation-structural rules. In Acoustic Communication in Birds (Kroodsma, D.E., Miller, E.H. and Ouellet, H., pp. 182212). Academic Press.

Ohtani, T., Matsuo, K., Kasai, K., Kato, T., \& Kato, N. (2005). Hemodynamic response to emotional memory recall with eye movement. Neuroscience Letters, 380(1), 75-79. https://doi.org/10.1016/j.neulet.2005.01.020

Okamoto, M., Dan, H., Sakamoto, K., Takeo, K., Shimizu, K., Kohno, S., Oda, I., Isobe, S., Suzuki, T., Kohyama, K., \& Dan, I. (2004). Three-dimensional probabilistic anatomical cranio-cerebral correlation via the international 10-20 system oriented for transcranial functional brain mapping. NeuroImage, 21(1), 99-111.

Perelman, P., Johnson, W. E., Roos, C., Seuánez, H. N., Horvath, J. E., Moreira, M. A. M., Kessing, B., Pontius, J., Roelke, M., Rumpler, Y., Schneider, M. P. C., Silva, A., O’Brien, S. J., \& Pecon-Slattery, J. (2011). A Molecular Phylogeny of Living Primates. PLOS Genetics, 7(3), e1001342. https://doi.org/10.1371/journal.pgen.1001342

Petrides, M., \& Pandya, D. N. (2002). Comparative cytoarchitectonic analysis of the human and the macaque ventrolateral prefrontal cortex and corticocortical connection patterns in the monkey. The European Journal of Neuroscience, 16(2), 291-310. https://doi.org/10.1046/j.1460-9568.2001.02090.x

Phelps, E. A., Lempert, K. M., \& Sokol-Hessner, P. (2014). Emotion and decision making: Multiple modulatory neural circuits. Annual Review of Neuroscience, 37, 263-287. https://doi.org/10.1146/annurev-neuro-071013-014119

Rolls, E. T. (2004). Convergence of sensory systems in the orbitofrontal cortex in primates and brain design for emotion. The Anatomical Record Part A: Discoveries in Molecular, Cellular, and Evolutionary Biology, 281A(1), 1212-1225. https://doi.org/10.1002/ar.a.20126

Sander, D., Grandjean, D., Pourtois, G., Schwartz, S., Seghier, M. L., Scherer, K. R., \& Vuilleumier, P. (2005). Emotion and attention interactions in social cognition: Brain regions involved in processing anger prosody. NeuroImage, 28(4), 848-858. https://doi.org/10.1016/j.neuroimage.2005.06.023

Schirmer, A., \& Kotz, S. A. (2006). Beyond the right hemisphere: Brain mechanisms mediating vocal emotional processing. Trends in Cognitive Sciences, 10(1), 24-30. https://doi.org/10.1016/j.tics.2005.11.009

Scholkmann, F., Spichtig, S., Muehlemann, T., \& Wolf, M. (2010). How to detect and reduce movement artifacts in near-infrared imaging using moving standard deviation and spline interpolation. Physiological Measurement, 31(5), 649-662. https://doi.org/10.1088/0967$3334 / 31 / 5 / 004$ 
Slocombe, K. E., Townsend, S. W., \& Zuberbühler, K. (2009). Wild chimpanzees (Pan troglodytes schweinfurthii) distinguish between different scream types: Evidence from a playback study. Animal Cognition, 12(3), 441-449. https://doi.org/10.1007/s10071-008-0204-x

Tak, S., Uga, M., Flandin, G., Dan, I., \& Penny, W. D. (2016). Sensor space group analysis for fNIRS data. Journal of Neuroscience Methods, 264, 103-112. https://doi.org/10.1016/j.jneumeth.2016.03.003

Team, R. (2020). RStudio: Integrated Development for $R$. RStudio. RStudio, Inc. https://rstudio.com/

Vergotte, G., Perrey, S., Muthuraman, M., Janaqi, S., \& Torre, K. (2018). Concurrent Changes of Brain Functional Connectivity and Motor Variability When Adapting to Task Constraints. Frontiers in Physiology, 9. https://doi.org/10.3389/fphys.2018.00909

Wagner, M., \& Watson, D. G. (2010). Experimental and theoretical advances in prosody: A review. Language and Cognitive Processes, 25(7-9), 905-945. https://doi.org/10.1080/01690961003589492

Wildgruber, D., Ethofer, T., Grandjean, D., \& Kreifelts, B. (2009). A cerebral network model of speech prosody comprehension. International Journal of Speech-Language Pathology, 11(4), 277-281. https://doi.org/10.1080/17549500902943043

Yang, H., Zhou, Z., Liu, Y., Ruan, Z., Gong, H., Luo, Q., \& Lu, Z. (2007). Gender difference in hemodynamic responses of prefrontal area to emotional stress by near-infrared spectroscopy. Behavioural Brain Research, 178(1), 172-176. https://doi.org/10.1016/j.bbr.2006.11.039

Zhang, D., Zhou, Y., \& Yuan, J. (2018). Speech Prosodies of Different Emotional Categories Activate Different Brain Regions in Adult Cortex: An fNIRS Study. Scientific Reports, 8(1), 218. https://doi.org/10.1038/s41598-017-18683-2 